\title{
A dog pedigree with familial medullary thyroid cancer
}

\author{
JIA-JING LEE ${ }^{1,2}$, CATHARINA LARSSON ${ }^{1}$, WENG-ONN LUI ${ }^{1}$, ANDERS HÖÖG ${ }^{2}$ and HENRIK VON EULER ${ }^{3}$ \\ Departments of ${ }^{1}$ Molecular Medicine and Surgery, ${ }^{2}$ Oncology-Pathology, Karolinska University \\ Hospital-Solna, Stockholm; ${ }^{3}$ Department of Small Animal Clinical Science, \\ Swedish University of Agricultural Sciences, Uppsala, Sweden \\ Received May 16, 2006; Accepted July 4, 2006
}

\begin{abstract}
Multiple endocrine neoplasia (MEN) is defined as concurrent neoplasia or hyperplasia in more than one endocrine gland. MEN is well known in humans and has also been reported in small animals. We report on a dog family of a mixed breed with Alaskan malamute as a major influence, where three members developed thyroid carcinomas and another dog had clinical signs mimicking the other three but without a confirmed diagnosis. The age of onset of the tumour was between 96-109 months. Clinical, biochemical and immunohistochemical examinations revealed that the affected individuals typically demonstrated symptoms including calcitonin positive thyroid cancer, hypothyroidism and chronic dermatitis. In addition, elevated serum calcium and multinodular adrenocortical hyperplasia were demonstrated in a single member. The diagnosis observed is similar to the familial form of medullary thyroid carcinoma (FMTC) in human. This is the first report of FMTC in dog. Up to $95 \%$ of FMTC and MEN2 is known to be caused by activating mutations in the RET gene. The dog Ret gene was analysed as a candidate in this pedigree. The complete dog Ret genomic sequence was predicted in silico. The lack of demonstratable Ret mutation suggests the involvement of alternative predisposing mutation in this pedigree. The unique occurrence of familial MTC makes this potentially an important model in further defining the genetic basis of MTC.
\end{abstract}

\section{Introduction}

Multiple endocrine neoplasia (MEN) is defined as concurrent neoplasia or hyperplasia in more than one endocrine gland. This is well known in humans, but has also been reported in small animals (Blackwood L, 15th ECVIM-CA Congress, Glasgow, UK, 2005). Two main types of MEN are recognised in human. MEN 1 is an autosomal dominant syndrome characterised by parathyroid hyperplasia, pituitary adenomas

Correspondence to: Dr Catharina Larsson, Department of Molecular Medicine and Surgery, Karolinska University HospitalSolna, CMM L8:01, SE-171 76 Stockholm, Sweden

E-mail: catharina.larsson@ki.se

Key words: dog, familial, FMTC, MEN, RET and endocrine pancreatic tumours. MEN2 is an autosomal dominant syndrome with a high degree of penetrance and variable clinical expressions. It exists in three clinically distinct forms: MEN2A, MEN2B and familial medullary thyroid carcinoma (FMTC) (1). MEN2A is the commonest subtype of MEN2 with a higher penetrance accounting for more than $90 \%$ of MEN2. MEN2A patients develop medullary thyroid carcinoma (MTC), pheochromocytoma and primary hyperparathyroidism. MEN2B, the most distinct and aggressive variant, occurs in $~ 5 \%$ of all cases of MEN2 (2). MEN2B shares the same clinical features as MEN2A except with an earlier onset age, absence of hyperparathyroidism and it has other developmental abnormalities such as decreased upper/ lower body ratio, marfanoid habitus, skeletal deformations, mucosal neuromas, ganglioneuromatosis of the intestinal tract, and myelinated corneal nerves (3-5). The third form of MEN2 is FMTC, with MTC as the only clinical feature. It is worth noting that MTC is the common feature of all these three variants of MEN2 and also occurs with a high penetrance.

MTC arises from the parafollicular or C cells of the thyroid, which have a unique ability to secrete calcitonin (CT). CT serves as the major clinical marker for the presence of MTC (6). The vast majority of MEN2 patients exhibit germ-line mutations in the RET (rearranged during transfection) protooncogene. Up to date, exons 8, 10, 11, 13, 14-16 of RET are hotspots for mutations identified to be associated with MEN2 syndrome in human (2).

Sporadic MTC has been previously reported in animals such as dog, red fox, bulls, rats, horse and ferret (7-12). Thyroid tumours are uncommon in the dog and account for $1-4 \%$ of all dog tumours (13). The most common thyroid tumours in dogs are of follicular origin (12). MTC is reported to correspond to $36 \%$ of the dog thyroid carcinomas and calcitonin is present in $70-100 \%$ of cases $(14,15)$.

For the first time we report and describe the occurrence of a familial thyroid carcinoma in dog. This led to the identification of dog Ret gene in silico, investigation and screening of this family for the occurrence of Ret mutations.

\section{Materials and methods}

Pedigree description. The pedigree consists of a mixed breed with Alaskan malamute as a major influence (Fig. 1, Table I). The father (1l:1) first presented in November 1998 with symptoms of itching and loss of luster to the hair coat. A skin biopsy showed severe para- and hyperkeratosis and chronic 


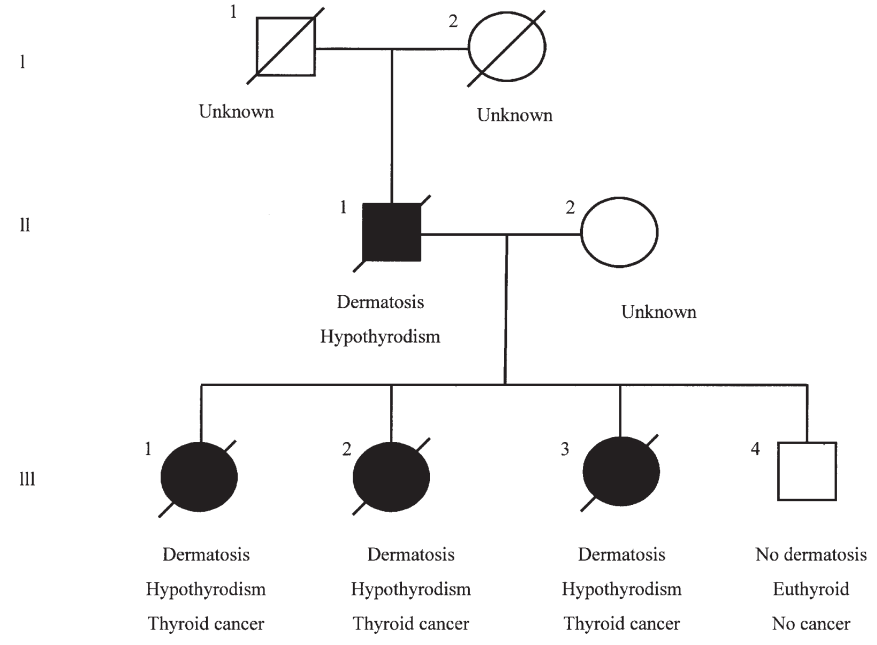

Figure 1. Pedigree of the dog family with thyroid carcinoma. Square denotes male and circle represents female. Affected individuals are highlighted black, whereas unaffected individuals remain white. A slash represents a deceased individual.

superficial pustular dermatitis, with no signs of sebaceous adenitis or pemphigus (analysed at REST associates, Cambridge, UK). Treatment with low dose prednisolon, antiparasitic drugs and cefalexin resulted in partial remission when the prednisolon dose was kept at $0.5 \mathrm{mg} / \mathrm{kg}$ SID. After discovering a severe hypothyroidism, levothyroxine $(0.03 \mathrm{mg} /$ $\mathrm{kg}$ SID) was added to the treatment regime resulting in an almost complete remission. In July 2003 the dermatological symptoms returned. The dog also exhibited subtle, abnormal signs of reduced activity, diminished appetite, tremor and muscle atrophy on the skull. Biochemical analysis showed anaemia and elevated ALP (assumingly due to steroid treatment). The dog was treated with clindamycin. In August 2003 the condition was dramatically worsened and the dog was treated for anaemia and acute hypovolemic shock. Despite supportive care the condition was not stabilised and the dog was euthanised. The owner declined autopsy.

Four months later the first out of four dogs (111:1) from the same litter was admitted to the Small Animal University Hospital, SLU with similar clinical symptoms as the father (11:1). The dog had chronic dermatitis, a 10x5 cm mass located in the subcutis adjacent to the cervical trachea and was in a semiconscious condition. Explorative surgery revealed a tumour mass that emanated from the thyroid and infiltrated the cervical musculature and regional lymph nodes. Since the condition was not improved and haemolytic anaemia was suspected, the dog was euthanised.

A few months later, the same owner entered the clinic with two littermates (111:2 and 111:3) who had developed similar symptoms as their sister. Thyroid ultrasound revealed highly vascularised masses in both dogs, which were biopsied and histopathologically diagnosed as thyroid cancer. The owner declined thyroidectomy. They also had prominent hypothyroidism. They were treated with oral levothyroxine $(0.03 \mathrm{mg} / \mathrm{kg}$ SID). However after two months, the treatment was discontinued since the dogs became hyperactive that could not be controlled by dose reduction. In April 2004 the second sister (111:2) was euthanised as the disease had progressed.
The youngest sister (111:3) was also euthanised in June 2005. The fourth littermate (1ll:4), a male, did not show any symptoms of the disease at clinical, biochemical or radiological examinations.

All dogs were thoroughly investigated with clinical status, biochemistry, imaging analyses and histopathology as detailed in Table I. Regular autopsy was performed in 111:1 and 111:2, and in 111:3 an extended autopsy was carried out.

Blood and tissue samples. The following clinical samples were collected for the study: peripheral blood samples from 111:1-4; paraffin-embedded tumour samples from primary tumours of 111:1-3, lymph node metastasis of 111:2; thyroid tumour biopsy of 111:3; a fresh frozen tumour sample from 111:2 and 111:3; paraffin-embedded autopsy samples of 111:1 including kidney, lung, liver and normal lymph node; and paraffin-embedded autopsy samples of 11l:3 from pituitary and adrenal. A normal thyroid from a healthy dog was analysed as control. All samples were obtained with informed consent and all treatments and sampling of dogs conformed to the decision of the Swedish Animal Ethics Committee (No. C 23/4).

Histopathological examination and immunohistochemistry. Paraffin-embedded sections of $4 \mu \mathrm{m}$ thickness were prepared from the primary thyroid tumour of (111:1, 11l:2 and 111:3), dewaxed, rehydrated and stained with haematoxylin and eosin as routine stain. Additional slides were pre-treated with citrate buffer at $\mathrm{pH} 6.0$ in a microwave oven for $20 \mathrm{~min}$. After rinsing, the endogenous peroxidase activity was blocked by treatment with $0.5 \%$ hydrogen peroxide for $30 \mathrm{~min}$. The sections were then rinsed, incubated with blocking serum (1\% bovine serum albumin) for $20 \mathrm{~min}$, and incubated with the primary antibody overnight at $4^{\circ} \mathrm{C}$. They were treated with the secondary antibody diluted 1:100 (anti-mouse $\mathrm{IgG}$ or anti-rabbit $\mathrm{IgG}$ with $<1 \%$ cross reactivity to $\operatorname{dog} \mathrm{IgG}$ ) for $30 \mathrm{~min}$, followed by rinsing and incubation with avidin-biotin-peroxidase complex (Vectastain Elite, Vector, UK) for $30 \mathrm{~min}$. The peroxidase reaction was developed using 3,3-diaminobenzidine for $6 \mathrm{~min}$. After counterstaining with Mayer's haematoxylin, the slides were dehydrated and mounted. Paraffin sections from thyroid collected from a normal dog were analysed in parallel as references. Staining for chromogranin A (1:150, NCL-Chrom, Novocastra, UK and 1:1500 and 1:3000, Yanaihara Institute, Shizuoka, Japan), chromogranin B (1:100, PH191, Binding Site, UK), synaptophysin (1:20, RM9111-S, Lab Vision, CA, USA) and RET (1:20, BAF482, R\&D Systems, MN, USA and 1:20, sc-167, Santa Cruz, CA, USA) were excluded because of negative results in the normal dog control indicating lack of cross reactivity to the dog epitopes. For calcitonin (1:1000, A576, DakoCytomation, Denmark), NSE (prediluted, Ventana, AZ, USA), PTH (1:3000, OP4, Novocastra), S-100 protein (1:2000, Z311, Dako), thyroglobulin (1:12000, A251, Dako) the expected staining results were obtained in normal dog thyroid.

mRNA in situ hybridisation for calcitonin. mRNA in situ hybridisation was performed on $4 \mu \mathrm{m}$ paraffin-embedded sections according to the protocol supplied by GeneDetect, Limited. The sections were hybridised with $200 \mathrm{ng} / \mathrm{ml}$ of anti- 
Table I. Clinical finding of the dog family.

\begin{tabular}{|c|c|c|c|c|c|}
\hline Parameter & 11:1 & 111:1 & 111:2 & 111:3 & 111:4 \\
\hline Diagnosis & Affected & Affected & Affected & Affected & Unaffected \\
\hline $\operatorname{Sex}(M / F)^{a}$ & $\mathrm{M}$ & $\mathrm{F}$ & $\mathrm{F}$ & $\mathrm{F}$ & M \\
\hline Age of disease onset & 96 months & 107 months & 109 months & 109 months & NA \\
\hline \multicolumn{6}{|l|}{ Clinical status } \\
\hline Fatigue & Yes & Yes & Yes & Yes & No \\
\hline Chronic dermatitis & Yes & Yes & Yes & Yes & No \\
\hline Weight loss & Yes & Yes & Yes & Yes & No \\
\hline Muscle atrophy & Yes & Yes & Yes & Yes & No \\
\hline Swollen neck & Yes & Yes & Yes & Yes & No \\
\hline Response to levothyroxine & Yes & Yes & Yes & Yes & NA \\
\hline Multifocal liver fibrosis & NA & Yes & Yes & Yes & No \\
\hline Hepatic necrosis & NA & Yes & Yes & Yes & No \\
\hline \multicolumn{6}{|l|}{ Hematology and biochemistry } \\
\hline Anemia & Yes & Yes & Yes & No & No \\
\hline Elevated ALP & Yes & Yes & Yes & Yes & No \\
\hline Hypothyroidism & Yes & Yes & Yes & Yes & No \\
\hline Positive $\mathrm{TgAb}$ & Yes & Yes & Yes & Yes & No \\
\hline Hypercalcemia & No & No & No & Yes & No \\
\hline \multicolumn{6}{|l|}{ Imaging performed } \\
\hline Neck ultrasound & NI & Yes & Yes (extended) & Yes & Yes \\
\hline Trunk X-ray & NI & Yes & Yes & Yes & Yes \\
\hline \multicolumn{6}{|l|}{ Histopathology } \\
\hline Autopsy performed & No & Yes & Yes & Yes (extended) & NA \\
\hline Skin biopsy performed & Yes & Yes & Yes & Yes & NA \\
\hline Tumour size & NI & $10 \times 5 \mathrm{~cm}$ & $6 \times 4 \mathrm{~cm}$ & $10 \times 4 \mathrm{~cm}$ & NA \\
\hline Thyroid carcinoma & NI & Yes & Yes & Yes & No \\
\hline Regional metastases & NI & Yes & Yes & Yes & NA \\
\hline \multicolumn{6}{|l|}{ Immunohistochemistry } \\
\hline NSE & NA & Yes & Yes & Yes & NI \\
\hline PTH & NA & No & No & No & NI \\
\hline $\mathrm{S} 100$ & NA & No & No & No & NI \\
\hline Calcitonin & NA & Yes & Yes & Yes & NI \\
\hline Thyroglobulin & NA & Yes & Yes & No & NI \\
\hline
\end{tabular}

${ }^{\mathrm{a}} \mathrm{M}$, male; F, female; NA, not applicable; NI, not investigated; ALP, alkaline phosphatase; TgAb, thyroglobulin autoantibody.

sense biotin-labelled (3'-end labelling) 48-mer oligonucleotide specific for dog calcitonin (5'-TCTCTGGCATCGGCTTCG GGGCTGAAACACCTGGCAAGAAAAGGGACA-3'). All sections were hybridised without probe and with antisense nonlabelled calcitonin probe as negative controls. The expected staining result was successfully performed on the normal dog thyroid, but not the tumour samples.

In silico analysis of dog Ret gene. To obtain full-length cDNA and genomic structure of the dog Ret gene, the human RET sequence (GenBank accession no. AJ243297) was used to perform a BLAST search in the HTGS database of the Dog
Genome (http://www.ncbi.nlm.nih.gov/genome/seq/CfaBlast. html). The Canis familaris BAC clone, RP81-410M10 (working draft Sequence, 7 ordered pieces, GenBank accession no. AC123973.2) has the highest alignment match with human RET. The sequence of this BAC clone was aligned with the partial dog Ret sequence (AF364316) for confirmation using BLAST (http://www.ncbi.nlm.nih.gov/blast/bl2seq/wblast2. cgi). Genomic dog Ret sequence, intron-exon borders and the coding region were determined by the GenScan prediction program (http://genes.mit.edu/GENSCAN.html). From the coding region, dog Ret amino acid sequence was deduced using ORF (Open Reading Frame) Finder (http://www.ncbi. 
Table II. Primers used for PCR amplification and sequencing of the dog Ret gene.

\begin{tabular}{|c|c|c|c|c|c|c|}
\hline \multirow{2}{*}{$\begin{array}{l}\text { Dog } \\
\text { Ret } \\
\text { target }\end{array}$} & \multirow{2}{*}{$\begin{array}{l}\text { Correspond. } \\
\text { human } \\
R E T\end{array}$} & \multicolumn{2}{|r|}{ Forward primer } & \multicolumn{2}{|r|}{ Reverse primer } & \multirow{2}{*}{$\begin{array}{l}\text { Product } \\
\text { size } \\
\text { (bp) }\end{array}$} \\
\hline & & Name & Sequence 5 '-3' & Name & Sequence 5'-3' & \\
\hline Ex 5 & Ex 2 & cfaRETex $2 \mathrm{~F}$ & CTGGGAGGTCTGCTCTGG & cfaRETex2R & GAGCCTCAGTGTGGGTGTG & 403 \\
\hline Ex 6 & Ex 3 & cfaRETex3F & CTGGACTTTCCACCAGCC & cfaRETex3R & GCCATACCTGGCATACAGC & 428 \\
\hline Ex 7 & Ex 4 & cfaRETex4F & TAGCTCACCCTGATTCCTGG & cfaRETex4R & ATAAATGGTGGACGGAGTGG & 385 \\
\hline Ex 9 & Ex 5 & cfaRETex $5 \mathrm{~F}$ & AAGTCTCAGGCTCCAGGGTC & cfaRETex5R & CTGTTGGAAGAGTGGACTGC & 330 \\
\hline Ex 10 & Ex 6 & cfaRETex6F & CAGTGGCATGGAGCAGTG & cfaRETex6R & GTTAAACAGAGTGCCCTCCC & 349 \\
\hline Ex 11 & Ex 7 & cfaRETex7F & TTCAGGCTGCCCAGAAAC & cfaRETex7R & CCCGTAGTTTTACCACACCC & 399 \\
\hline Ex 12 & Ex 8 & cfaRETex $8 \mathrm{~F}$ & ATGCTGGCTGGACACTGG & cfaRETex $8 \mathrm{R}$ & ACTCAGCCACCATTACCCC & 247 \\
\hline Ex 13 & Ex 9 & cfaRETex9F & CCTTCTGGCTCTGGCAGTC & cfaRETex9R & CATTGGCTGGCTACCCCTAC & 248 \\
\hline Ex 14 & Ex 10 & cfaRETex $10 \mathrm{~F}$ & GGGTGGTCCTGGTTTGTC & cfaRETex10R & GGGTTTCACCTGCTTTCC & 282 \\
\hline Ex 15 & Ex 11 & cfaRETex11F & GCCACAGCAGCCTTCCAG & cfaRETex11R & CCCACACCTCCCAGCTTC & 429 \\
\hline Ex 16 & Ex 12 & cfaRETex $12 \mathrm{~F}$ & АСССТСАССААСТТТСТССС & cfaRETex12R & CTTTAGAGGGCCCTAGGCTG & 304 \\
\hline Ex 17 & Ex 13 & cfaRETex13F & CCAGGGATGGTCACAAGAAG & cfaRETex13R & AAGCAGCCCAGAACCCTTAG & 239 \\
\hline Ex 18-19 & Ex 14-15 & cfaRETex14/15F & TTGGTAGGATACAGAGCCTGC & cfaRETex14/15R & CTACCCACTGCAGGAACCC & 605 \\
\hline Ex 20 & Ex 16 & cfaRETex $16 \mathrm{~F}$ & CCCAGGGTGTTGTCAGAGTG & cfaRETex16R & GGTCCCTGCCATTTGCTG & 195 \\
\hline Ex 21 & Ex 17 & cfaRETex17F & ACATATCGTCTGCTGAGGGG & cfaRETex17R & GGAGCAGGGAGGGAATGAG & 278 \\
\hline Ex 22 & Ex 18 & cfaRETex $18 \mathrm{~F}$ & TGTGATTGGGCAATCCTTG & cfaRETex18R & GAACTGAGGTGAGGCTGGAG & 236 \\
\hline Ex 23 & Ex 19 & cfaRETex19F & AAGGGGCGAGTCCAGTTG & cfaRETex19R & GAGAGCTTTGGTTTCATTCAGA & 320 \\
\hline Ex 24 & Ex 20 & cfaRETex20F & TTTGCCAAGGCCTTACTGTC & cfaRET3utrR & GCAGACTTTCCATTCTCGGC & 298 \\
\hline
\end{tabular}

Correspond., corresponding.

nlm.nih.gov/gorf/gorf.html). The predicted dog Ret amino acid was compared to its human counterpart using ClustalW (http://www.ebi.ac.uk/clustalw) sequence alignment program.

\section{Mutational screening of Ret}

Genomic DNA extraction. Ten milliliters of blood from individual 111:2, 111:3, 111:4, normal control and an unrelated dog were used for genomic DNA extraction using Wizard ${ }^{\circledR}$ Genomic Purification Kit (Promega). Genomic DNA was also extracted from tumour tissue of individual 11l:2 using Qiagen DNA Mini Kit.

DNA amplification. Primers used in amplification of exons 5-7 and 9-24 corresponding to exons 2-20 of its human counterpart are described in Table II. Briefly, the PCR reaction was performed in GeneAmp ${ }^{\circledR}$ PCR System 9700 (PE, Applied Biosystems, Foster City, CA) employing 50 ng genomic DNA in a 50- $\mu 1$ volume containing $1 \mathrm{U}$ AmpliTaq Gold Polymerase (Applied Biosystems), $200 \mu \mathrm{M}$ dNTP, $2.5 \mu \mathrm{M}$ of $\mathrm{MgCl}_{2}$ and 25 pmol of each specific primer (Proligo, France). Cycling conditions to generate PCR products included an initial phase of $10 \mathrm{~min}$ at $96^{\circ} \mathrm{C}$, followed by 35 cycles of $30 \mathrm{sec}$ at $96^{\circ} \mathrm{C}$, $45 \mathrm{sec}$ at $65^{\circ} \mathrm{C}$ and $1 \mathrm{~min}$ at $72^{\circ} \mathrm{C}$ and a final extension step of $10 \mathrm{~min}$ at $72^{\circ} \mathrm{C}$.

SSCP and sequencing analysis. The presence or absence of variation of each amplicon was assessed by single strand conformation polymorphism (SSCP) analysis using GeneGel SSCP Starter Kit (Amersham Biosciences). The nucleotide sequences of amplified products that show an abnormal electrophoretic mobility on SSCP analysis and those amplicons that were reported as mutation hot-spots in human MEN2 were directly sequenced using an automated system employing BigDye $^{\circledR}$ Terminator v3.1 (Applied Biosystems) and analysed on an ABI3700 sequencer (PE, Applied Biosystems).

\section{Results}

Clinical presentations. Four affected and one clinically unaffected members of the pedigree were studied. The chronic hyperplastic dermatosis presented as sparse hair coat with alopecia (bilateral, symmetric and non-pruritic), mainly affecting the flank, neck, posterior thighs and tail (rat-like tail) (illustrated for 111:3 in Fig. 2A). From the histopathological examination of the skin biopsy, the hair coat was found to have hyperpigmentation, hypertrichosis, hyperkeratosis, acanthosis and distortion with large sebaceous and dilated sweat glands. The thickened and puffy skin due to oedema produced exaggerated skin folds on forehead and neck. This caused anxiety with discomfort, hyperactivity and change in behaviour. Slow hair growth with few hair follicles in the anagen phase, slow wound healing and easy bruising were also observed. In all the affected dogs, the chronic dermatosis included alopecia, hyperpigmentation, pyodermia and itching. 


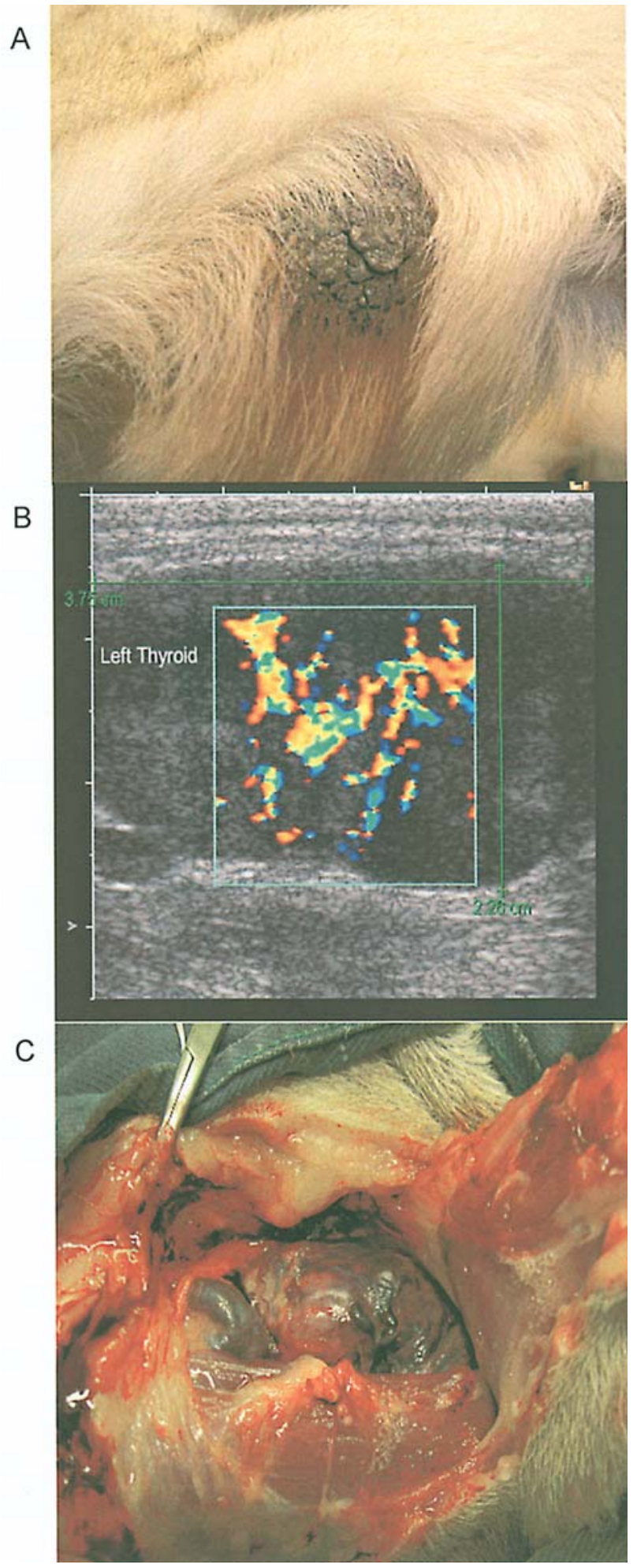

Figure 2. (A), Presentation of skin dermatosis in dog 111:3. The hair coat was sparse in a bilateral and symmetrical pattern with alopecia. (B), Color doppler ultrasound imaging of dog 11l:3 showing the intensive vascularisation of the entire thyroid cancer. (C), Thyroid tumour in situ of dog 111:3. Note the large, aberrant vessels around the tumour.

Symptoms of fatigue, weight loss, muscle atrophy, hypothyroidism and neck oedema were also observed in individuals 11:1, 111:1, 111:2 and 111:3. Biochemically, these dogs were found to have severe anaemia, hypothyroidism with low free and total serum T4, elevated TSH and positive thyroglobulin autoantibody (TgAb). High serum calcium was noted in one
Table III. Genomic structure of the dog Ret gene corresponding to the human cDNA (AJ243297).

\begin{tabular}{|c|c|c|c|c|}
\hline & $\begin{array}{l}\text { Sequence of Ret } \\
\text { Splice junctions }\end{array}$ & $\begin{array}{l}\text { Dog Ret } \\
\text { Size (bp) }\end{array}$ & $\begin{array}{c}\text { Human } \\
\text { RET } \\
\text { Size (bp) }\end{array}$ & $\begin{array}{c}\text { Correspond. } \\
\text { to human } \\
R E T \text { exon/ } \\
\text { intron no. }\end{array}$ \\
\hline Ex 1 & GCAAGgtaaaacagc & ${ }^{\mathrm{a}} 143+\mathrm{UTR}$ & 253 & $\mathrm{NC}$ \\
\hline Int 1 & ccetgecccagCCСТC & 2898 & 23127 & $\mathrm{NC}$ \\
\hline Ex 2 & GTGCTgtgcgtggct & 122 & & $\mathrm{NC}$ \\
\hline Int 2 & gtgtcttcagCTGCC & 449 & & $\mathrm{NC}$ \\
\hline Ex 3 & AGGAGgtcagccetg & 98 & & $\mathrm{NC}$ \\
\hline Int 3 & tccttcccagTCCAG & 556 & & $\mathrm{NC}$ \\
\hline Ex 4 & AGCAGgtggggccaa & 25 & & $\mathrm{NC}$ \\
\hline Int 4 & ctttctgcagCTCCA & 1948 & & $\mathrm{NC}$ \\
\hline Ex 5 & CCGCAgtaagggagc & 267 & 264 & Ex 2 \\
\hline Int 5 & ctctgtctacagATGGC & 798 & 1619 & Int 2 \\
\hline Ex 6 & AGAAGgtgagtgctg & 288 & 288 & Ex 3 \\
\hline Int 6 & tggtgtgtagGTGAG & 2158 & 2322 & Int 3 \\
\hline Ex 7 & AGGAGgtgcetgtct & 245 & 242 & Ex 4 \\
\hline Int 7 & ttctcctaagGGACC & 879 & 1182 & Int 4 \\
\hline Ex 8 & CACAGgtcagtctgt & 168 & & $\mathrm{NC}$ \\
\hline Int 8 & ttgtctgcagGGCAC & 140 & & $\mathrm{NC}$ \\
\hline Ex 9 & CTACAgtaagagggg & 196 & 196 & Ex 5 \\
\hline Int 9 & ccacccacagGGCTG & 1296 & 2459 & Int 5 \\
\hline Ex 10 & CCCAGgtgagcetgc & 200 & 200 & Ex 6 \\
\hline Int 10 & ccatgcctagATTGG & 2060 & 1976 & Int 6 \\
\hline Ex 11 & GACATgtgagtgeca & 259 & 259 & Ex 7 \\
\hline Int 11 & ccacctgcagATGTG & 711 & 633 & Int 7 \\
\hline Ex 12 & CAAAGgtaggeccca & 126 & 126 & Ex 8 \\
\hline Int 12 & tgtattatagGGATC & 544 & 628 & Int 8 \\
\hline Ex 13 & CCTCCgtaagcaacc & 111 & 111 & Ex 9 \\
\hline Int 13 & ctgtcaccagGAGGT & 540 & 592 & Int 9 \\
\hline Ex 14 & CCAGGgtgagtgggt & 123 & 120 & Ex 10 \\
\hline Int 14 & ctgcccacagACCCA & 930 & 804 & Int 10 \\
\hline Ex 15 & TCCCGgtgaggctct & 257 & 257 & Ex 11 \\
\hline Int 15 & ccccacatagGAGGA & 3064 & 1847 & Int 11 \\
\hline Ex 16 & GAAAGgtacctgtcc & 148 & 148 & Ex 12 \\
\hline Int 16 & tgcatttcagAGAAT & 1485 & 1641 & Int 12 \\
\hline Ex 17 & GGATGgtaaggecgg & 108 & 108 & Ex 13 \\
\hline Int 17 & ctgcccccagGGCCA & 994 & 1050 & Int 13 \\
\hline Ex 18 & TGAAGgtgtgtgcca & 215 & 215 & Ex 14 \\
\hline Int 18 & ttccetgcagCTTGT & 87 & 335 & Int 14 \\
\hline Ex 19 & GCAAGgtaccaggtc & 123 & 123 & Ex 15 \\
\hline Int 19 & ccctttatagGGTCG & 1981 & 1742 & Int 15 \\
\hline Ex 20 & GATGTgtaagtgtgg & 71 & 71 & Ex 16 \\
\hline Int 20 & ctccccttagGTGGT & 1277 & 1654 & Int 16 \\
\hline Ex 21 & GAGATgtgagcaggc & 138 & 138 & Ex 17 \\
\hline Int 21 & ttcccgccagGTATG & 1110 & 1074 & Int 17 \\
\hline Ex 22 & ACAGAgtgagtacctgg & 100 & 100 & Ex 18 \\
\hline Int 22 & tctcttccagGACTA & 1510 & 1592 & Int 18 \\
\hline Ex 23 & TCTAGcaacgttgtg & 180 & 148+UTR & Ex 19 \\
\hline Int 23 & tcatttttaggGCATG & 1811 & 1389 & Int 19 \\
\hline Ex 24 & GTTAAcatttctttg & ${ }^{\mathrm{a}} 158+\mathrm{UTR}$ & 1390 & Ex 20 \\
\hline
\end{tabular}

Ex, exon; Int, intron. NC, exon/intron sequences not corresponding to the human exon/intron sequences. Uppercase and lowercase letters, exonic and intronic sequences, respectively. ${ }^{\text {a } 5}$ '-end of exon 1 and 3'-end of exon 24 have not yet been determined. Correspond., corresponding. 


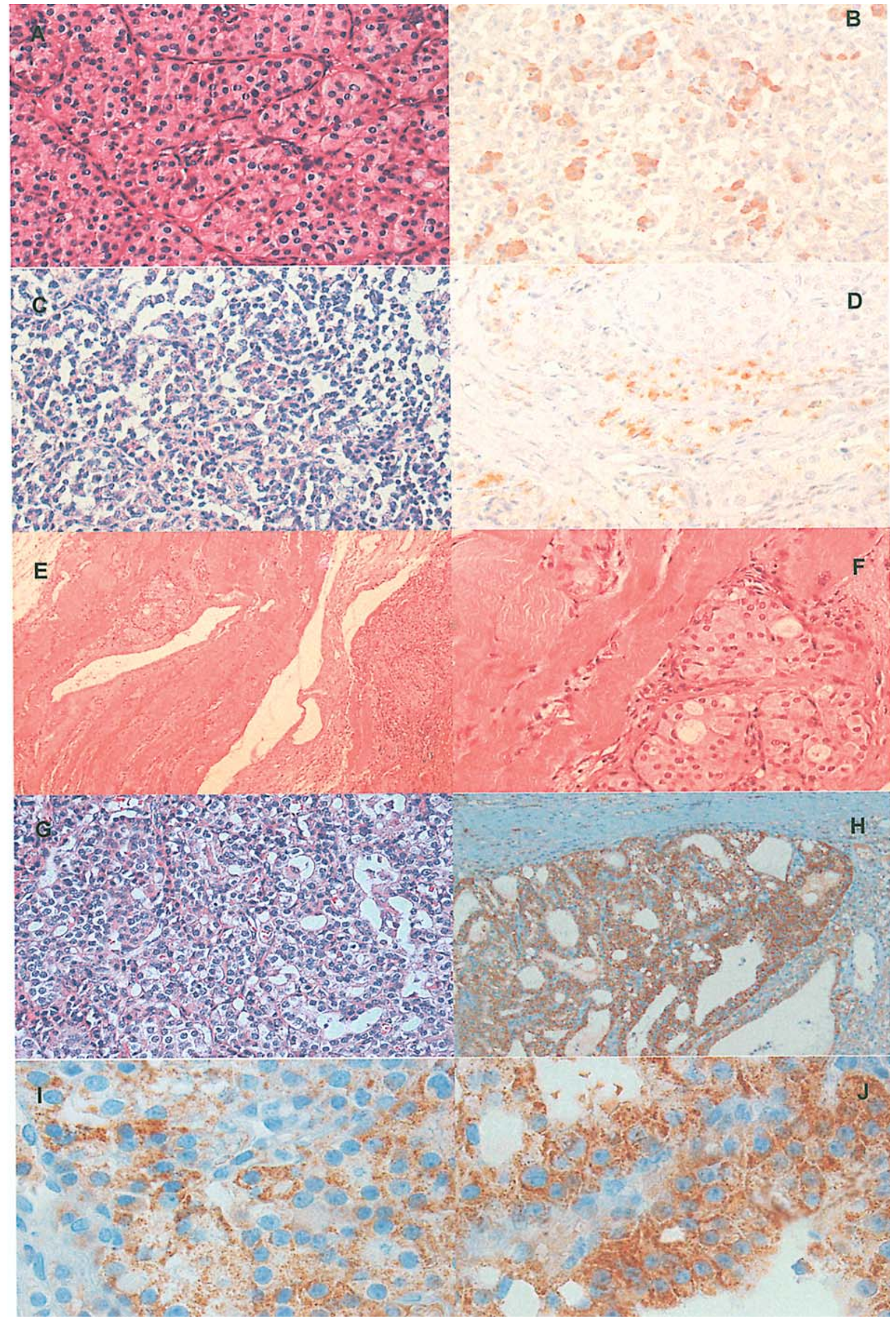

Figure 3. (A), H\&E staining of the thyroid from 11l:1 (x180). (B), Immunohistochemical (IHC) staining for calcitonin of the thyroid cancer from 111:1. Cells with positive expression stained brown (x180). (C), H\&E staining of the thyroid cancer from 111:2 (x180). (D), IHC expression of calcitonin in the thyroid cancer from 111:2 (x180). (E and F), H\&E staining of the thyroid cancer infiltrating the adjacent muscle from 111:2 ( $x 45$ and $x 180)$. (G), H\&E staining of the thyroid cancer from 111:3 (x180). (H, I and J), IHC expression of calcitonin in the thyroid tumour of 111:3 (x90, x180 and x440). 


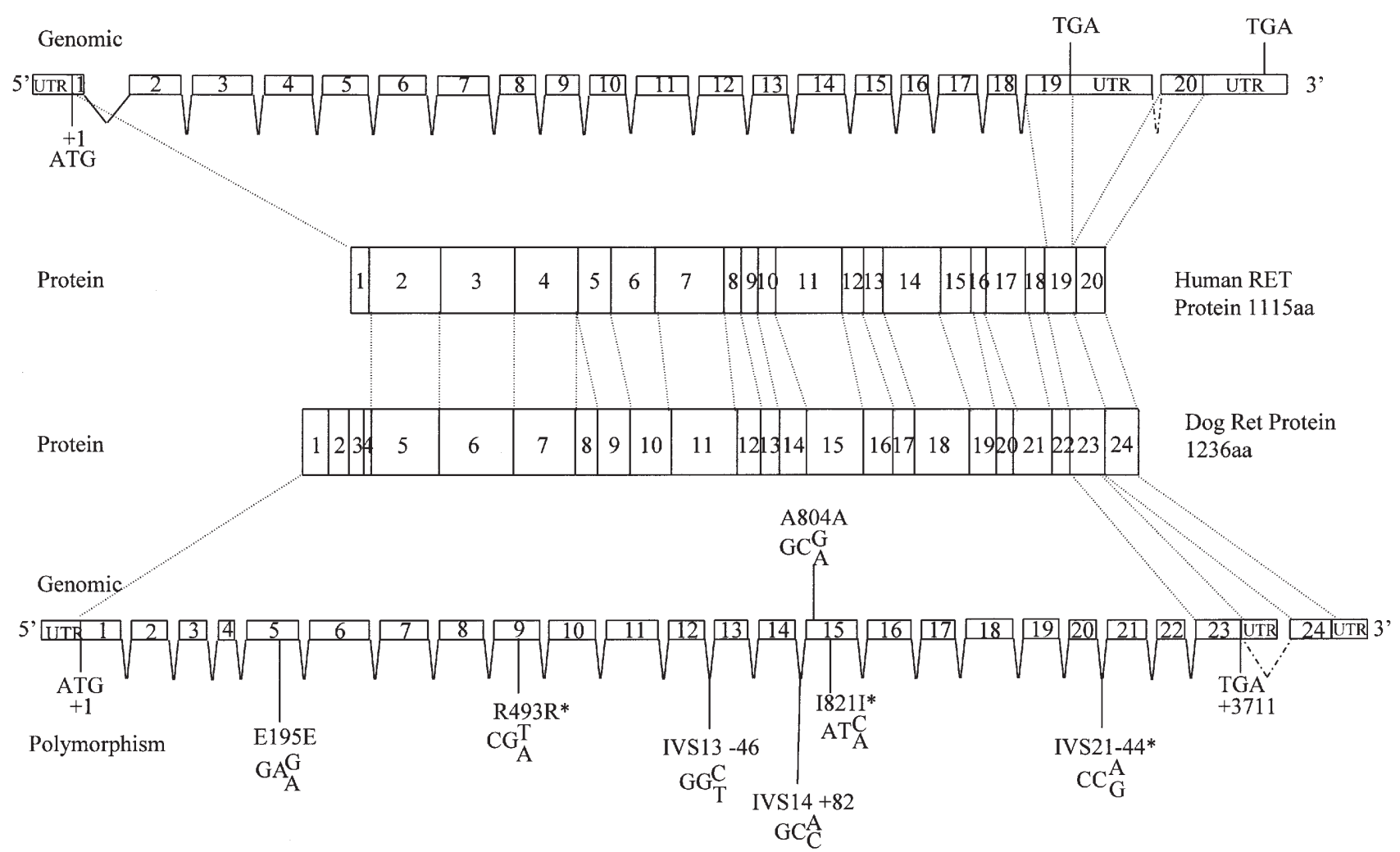

Figure 4. Genomic organisation of dog and human RET. Sequence variants detected in the dog Ret and their respective encoding proteins are shown at the bottom. Variants found in the pedigree are denoted as asterisk.

of the affected dogs only (111:3). Neck ultrasound typically showed bilateral highly vascularised tumour masses (Fig. 2B). No other tumour was found at trunk imaging.

Autopsy was performed in 111:1, 111:2 and 111:3. This confirmed that the tumour emanated from the thyroid (Fig. 2C). 111:1 and 111:2 consisted of a differentiated, thyroid carcinoma with metastases to the cervical lymph nodes. A prominent oedema was present in the pharynx and subcutis in the headneck region. Finally the dogs had multi-focal liver fibrosis and hepatic autolytic changes. An extended autopsy was also performed for 111:3 that showed similar findings as in 111:1. The thyroid carcinoma exhibited infiltrative growth into surrounding tissues and vessels, and in addition metastases were found in the thymus. Microscopically, the tumour was composed of a mixture of acinar and compact growing epithelial cells. No pheochromocytoma or pituitary adenoma could be found. However, unilateral multifocal adrenocortical hyperplasia was observed.

Histopathology and immunohistochemical findings. All three tumours examined (111:1-3) showed similar histopathology and immunostaining results (Fig. 3; Table I). At histopathological examination, the tumours exhibited generally compact cell growth pattern or follicular pattern with intramuscular infiltration. The medium sized tumour cells had light to eosinophilic cytoplasm with round or slightly elongated nuclei. At immunohistochemistry all tumours were clearly positive for NSE and negative for PTH and S-100 protein. Various proportions of the tumour cells were positive for calcitonin. To confirm the accuracy of the calcitonin antibody, sections of normal dog thyroid were analysed with a small portion of
C-cells expressing calcitonin. The mRNA in situ demonstrated calcitonin expression in C-cells from normal thyroid (data not shown). Immunohistochemistry with the calcitonin antibody on dog thyroid control sections produced a similar pattern of C-cells staining. The tumours also showed positive expression of thyroglobulin in a subset of tumour cells by immunohistochemistry except for 111:3, which was negative. Based on these findings, the tumours were classified as highly malignant, differentiated thyroid carcinoma, possibly of medullary or mixed follicular-medullary subtype. As differential diagnoses, thyroid paraganglioma, parathyroid carcinoma and follicular thyroid carcinoma were considered.

Dog Ret gene bioinformatics. A full-length cDNA and genomic structure of the dog Ret gene (GenBank submission BK005621) was determined by using the computational bioinformatics based on the human RET sequence (AJ243297). Analysis by GenScan (MIT, USA) on the sequence derived from dog BAC clone RP81-410M10 (GenBank accession no. AC123973.2) predicted 23 exons in the dog Ret gene, giving a 3711-bp of open reading frame (ORF). A separate putative 24th exon of the dog Ret was predicted and matched with the human $R E T$ exon 20 by aligning with its human counterpart. The predicted structure and exon-intron boundaries of dog Ret coincides with that of human RET as predicted by alignment of RET cDNA against the human genome sequence (Table III, Fig. 4), showing $86 \%$ identity in the nucleotide level. Alignment of cDNA RET orthologues of both species showed that the putative exons 5-7 and 9-24 in the dog Ret correspond to exons 2-4 and 5-20 in the human RET respectively. A 1236 aa dog Ret protein was predicted (Dog isoform A) and was 

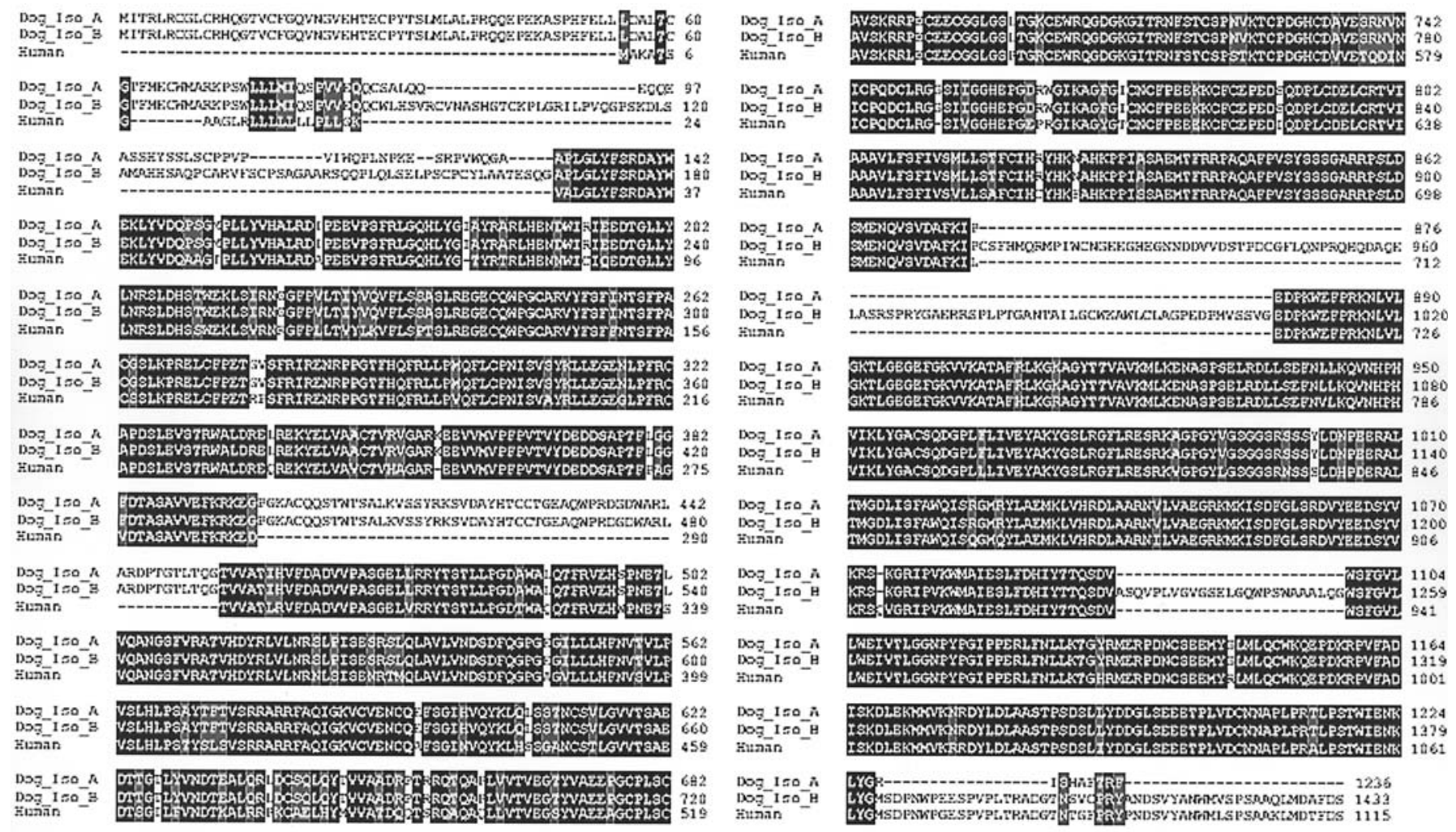

Figure 5. Alignment of the predicted dog Ret proteins (two isoforms) with human RET protein. Identical amino acids are highlighted in black and similarity amino acids are highlighted in grey. Dog_Iso_A denotes dog Ret isoform A and Dog_Iso_B denotes dog Ret isoform B.

aligned with its human counterpart (1115 aa), showing 83\% identity (Fig. 5).

Ret mutational screening. We screened individuals 111:1, 11l:2, 111:3 and 111:4 for mutations in exons 12-21 of the dog Ret gene (corresponding to human exons 8-17), where all the known MEN2 and MTC related mutations are located by sequencing analysis. The screening of other exons that corresponded to other human RET exons was carried out by SSCP. Sequencing was performed on all variations observed by SSCP. Three variations were present in these individuals of the family but did not segregate with the disease. Four other variations were only observed in an unrelated dog (Fig. 4), which was included in the screening as comparison.

\section{Discussion}

We report a dog family where four members developed thyroid carcinomas in a pattern suggesting dominant inheritance of autosomal or X-linked type. From the clinical, histopathological and biochemical examinations, the affected individuals demonstrated symptoms including calcitonin positive thyroid cancer, hypothyroidism, chronic dermatitis, and in one case elevated serum calcium levels. These findings suggest a familial form of medullary thyroid carcinoma similar to human FMTC. However, the observation of multinodular hyperplasia in the adrenal cortex of dog 11l:3 at autopsy would suggest a form of hereditary multiple endocrine neoplasia (MEN) not restricted to FMTC. On the other hand, this dog suffered from the most progressed and long-standing MTC. Therefore the observed changes in the adrenal cortex could also represent secondary alterations due to physical and hormonal stress.

The finding of familial MTC prompted the screening for constitutional Ret mutation. To date, only a partial dog Ret cDNA sequence spanning 2 exons was reported (GenBank AF364316) (16). In this study, we have predicted the complete dog Ret genomic sequence (BK005621), which is $\sim 33 \mathrm{~kb}$ long giving a 3711-bp of ORF (23 exons) and a predicted 1236 aa protein (isoform A). According to the human Ret protein sequence, there are two major isoforms, with a smaller isoform encoded from exons 2 to 19 and a longer isoform encoded from exons 2 to 20 of a different transcript. From the latest completed first draft dog genome sequence, we found another isoform (isoform B), predicted using GenScan based on the draft genomic sequence (UCSC genome browser database of dog). Isoform B was predicted to span 1433 aa encoded from 4302 bp of cDNA (24 exons). In addition recently there was a predicted full length dog Ret cDNA in the Ensembl Database (ENSCAFG00000007076), this predicted sequence spans 1944 bp (12 exons) with 413 aa translated. This annotated sequence (ENSCAFG00000007076) was aligned and revealed to be within our predicted sequence (data not shown). The $\operatorname{dog}$ isoforms A and B share high similarity and both have a human counterpart. Notably the entire coding parts of the hot spot exons were entirely screened.

We explored the possible involvement of Ret in this dog family due to the established causative role of RET in inherited MTC in human. All exons of the annotated dog Ret gene were explored either by SSCP or/and sequencing, except exons 1-4 and 8 as these exons were found not to be corresponding to 
human RET. The variations found did not segregate with the disease in this family, and we therefore conclude that these are polymorphisms of no clinical relevance to the phenotype. From a comparative genetic point of view, the familial MTC in this family does not share the genetic etiology commonly underlying phenotype in human. Therefore, mutations in the same dog Ret outside the human hotspots as well as mutations in other genes should be considered.

Until date $\sim 5 \%$ of human MEN2 kindreds have been reported to be without the RET genetic alterations (17). It was recently suggested that alterations in the succinate dehydrogenase D gene (SDHD) was responsible for familial non-RET C-cell hyperplasia, which is associated with MTC (18). While it is presently unclear if $S D H$ genes are causative genes for non-RET C-cell hyperplasia, the possibility of other genes in the development of tumourigenesis in non-RET familial MEN2 syndromes could not be excluded (19). The absence of demonstratable Ret mutation in this study, further suggests the possibility of additional genes involved in the tumourigenesis of MTC.

All three examined dogs exhibited similar pathology of their thyroid cancers. By immunohistochemistry calcitonin (C-cells) were found expressed in the tumour tissues of all three cases, in agreement with a diagnosis of medullary thyroid carcinoma. Serum measurement of calcitonin levels would be optimal to confirm the diagnosis. However, no such assay is presently available for dog species (20). Given the prominent tumour vascularity, the thyroglobulin positivity in two cases and the single case of dog with hypercalcemia, we have also considered the following differential diagnoses: thyroid paraganglioma, parathyroid carcinoma and follicular thyroid carcinoma. However, these alternative diagnoses were considered highly unlikely because of the negative staining results for S-100 protein (tumour marker for paraganglioma) and PTH (tumour marker for parathyroid) and positive staining for calcitonin (a tumour marker for MTC).

Although thyroglobulin was found to be expressed in two tumour tissues (11l:1 and 111:2), no thyroglobulin was expressed in the tumour tissues of 111:3. This picture would exclude both follicular thyroid carcinoma and follicular carcinoma of mixed follicular-medullary subtype as differentiated diagnosis. It is likely that the timing of the tissue preservation attributed to the positive staining for thyroglobulin. In this case, the tumour tissues from 111:3 were well preserved due to immediate fixation after autopsy, whereas there was a delayed in fixing the tumour tissues from 1ll:1 and 11l:2. It has also been previously demonstrated in human tumour tissues that thyroglobulin staining could be easily diffused and thus creating a false positive staining in immunohistochemistry in less well-preserved tissues (unpublished data). The mixed staining of thyroglobulin in medullary carcinomas in dogs has been previously observed (12). It was suggested that the expression of thyroglobulin observed was due to the pre-existing thyroid tissues included in the tumour and not from the tumour tissue itself. Two studies in human have reported MTCs concurrently producing calcitonin and thyroglobulin $(21,22)$.

Although most of the genetic basis of MEN syndromes has been elucidated in humans, the molecular pathogenesis of new familial MEN-type tumours remain to be identified. There are ongoing studies using rat as a model to define the genetic mechanisms of a newly identified MEN-like syndrome called MEN X $(23,24)$. MEN X is characterised by inborn cataract and multiple tumours affecting the neuroendocrine system and the disease gene has been mapped and the data demonstrated has excluded the involvement of Ret. The unique occurrence of familial MTC makes this potentially an important model in further defining the genetic basis of MTC.

\section{Acknowledgements}

We thank the Department of Clinical Radiology for supplying the ultrasound image, Department of Biomedical Sciences and Veterinary Public Health, Division of Pathology, Pharmacology and Toxicology and the Laboratory of Pathology at SVA (National Veterinary Institute of Sweden) for supplying the paraffin blocks and Department of Veterinary Anatomy and Physiology for providing the specimens of normal dog thyroid gland. This study was supported by the Swedish Cancer Foundation, the Stockholm County Council, the Swedish Research Council, the Stockholm Cancer Society and the Swedish Society of Medicine.

\section{References}

1. Mulligan LM, Marsh DJ, Robinson BG, et al: Genotype-phenotype correlation in multiple endocrine neoplasia type 2: report of the International RET Mutation Consortium. J Intern Med 238: 343-346, 1995.

2. Peczkowska $M$ and Januszewicz A: Multiple endocrine neoplasia type 2. Fam Cancer 4: 25-36, 2005.

3. Bachelot A, Lombardo F, Baudin E, Bidart JM and Schlumberger M: Inheritable forms of medullary thyroid carcinoma. Biochimie 84: 61-66, 2002.

4. Hansford JR and Mulligan LM: Multiple endocrine neoplasia type 2 and RET: from neoplasia to neurogenesis. J Med Genet 37: 817-827, 2000.

5. Hoff AO, Cote GJ and Gagel RF: Multiple endocrine neoplasias. Annu Rev Physiol 62: 377-411, 2000.

6. Massoll N and Mazzaferri EL: Diagnosis and management of medullary thyroid carcinoma. Clin Lab Med 24: 49-83, 2004

7. Boorman GA, van Noord MJ and Hollander CF: Naturally occurring medullary thyroid carcinoma in the rat. Arch Pathol 94: 35-41, 1972.

8. Fox JG, Dangler CA, Snyder SB, Richard MJ and Thilsted JP: C-cell carcinoma (medullary thyroid carcinoma) associated with multiple endocrine neoplasms in a ferret (Mustela putorius). Vet Pathol 37: 278-282, 2000

9. Hirayama K, Kagawa Y, Nihtani K and Taniyama H: Thyroid C-cell carcinoma with amyloid in a red fox (Vulpes vulpes schrenchki). Vet Pathol 36: 342-344, 1999.

10. Kuwamura M, Shirota A, Yamate J, Kotani T, Ohashi F and Sakuma S: C-cell adenoma containing variously sized thyroid follicles in a horse. J Vet Med Sci 60: 387-389, 1998.

11. Ljungberg $\mathrm{O}$ and Nilsson PO: Hyperplastic and neoplastic changes in ultimobranchial remnants and in parafollicular (C) cells in bulls: a histologic and immunohistochemical study. Vet Pathol 22: 95-103, 1985.

12. Patnaik AK and Lieberman PH: Gross, histologic, cytochemical, and immunocytochemical study of medullary thyroid carcinoma in sixteen dogs. Vet Pathol 28: 223-233, 1991.

13. Page R: Tumours of the endocrine system. In: Small Animal Clinical Oncology. Withrow SJ and Macewen EG (eds). 3rd edition. W.B. Saunders, Philadelphia, PA, pp418-444, 2001.

14. Carver JR, Kapatkin A and Patnaik AK: A comparison of medullary thyroid carcinoma and thyroid adenocarcinoma in dogs: a retrospective study of 38 cases. Vet Surg 24: 315-319, 1995

15. Ramos-Vara JA, Miller MA, Johnson GC and Pace LW: Immunohistochemical detection of thyroid transcription factor-1, thyroglobulin, and calcitonin in canine normal, hyperplastic, and neoplastic thyroid gland. Vet Pathol 39: 480-487, 2002. 
16. Popsueva A, Poteryaev D, Arighi E, Meng X, Angers-Loustau A, Kaplan D, Saarma M and Sariola H: GDNF promotes tubulogenesis of GFRalpha1-expressing MDCK cells by Src-mediated phosphorylation of Met receptor tyrosine kinase. J Cell Biol 161: 119-129, 2003.

17. Santoro M, Carlomagno F, Romano A, et al: Activation of RET as a dominant transforming gene by germline mutations of MEN2A and MEN2B. Science 267: 381-383, 1995.

18. Lima J, Teixeira-Gomes J, Soares P, Maximo V, Honavar M, Williams D and Sobrinho-Simoes M: Germline succinate dehydrogenase subunit $\mathrm{D}$ mutation segregating with familial non-RET C cell hyperplasia. J Clin Endocrinol Metab 88: 4932-4937, 2003.

19. Cascon A, Cebrian A, Pollan M, Ruiz-Llorente S, MonteroConde C, Leton R, Gutierrez R, Lesueur F, Milne RL, Gonzalez-Albarran O, Lucas-Morante T, Benitez J, Ponder BA and Robledo M: Succinate dehydrogenase D variants do not constitute a risk factor for developing $\mathrm{C}$ cell hyperplasia or sporadic medullary thyroid carcinoma. J Clin Endocrinol Metab 90: 2127-2130, 2005.
20. Hazewinkel HA, Schoenmakers I, Pelling D, Snijdelaar M, Wolfswinkel J and Mol JA: Biological potency and radioimmunoassay of canine calcitonin. Domest Anim Endocrinol 17: 333-344, 1999.

21. Holm R, Farrants GW, Nesland JM, Sobrinho-Simoes M, Jorgensen OG and Johannessen JV: Ultrastructural and electron immunohistochemical features of medullary thyroid carcinoma. Virchows Arch A Pathol Anat Histopathol 414: 375-384, 1989.

22. Holm R, Sobrinho-Simoes M, Nesland JM, Gould VE and Johannessen JV: Medullary carcinoma of the thyroid gland: an immunocytochemical study. Ultrastruct Pathol 8: 25-41, 1985.

23. Fritz A, Walch A, Piotrowska K, Rosemann M, Schaffer E, Weber K, Timper A, Wildner G, Graw J, Hofler H and Atkinson MJ: Recessive transmission of a multiple endocrine neoplasia syndrome in the rat. Cancer Res 62: 3048-3051, 2002.

24. Piotrowska K, Pellegata NS, Rosemann M, Fritz A, Graw J and Atkinson MJ: Mapping of a novel MEN-like syndrome locus to rat chromosome 4. Mamm Genome 15: 135-141, 2004.

\section{Supplementary}

We have read the article by Sako et al (J Comp Pathol 133: 155-163, 2005) about chromogranin A in neuroendocrine carcinomas of dogs. The antibody used in that article was purchased from a company in Japan (Yanaihara Institute, Shizuoka, Japan). This antibody was tested on normal dog thyroid with an abundance of calcitonin cells (Supplementary Fig. 1A). Pretreatment and dilution of the antibody was the same as in the article by Sato et al. The dilution 1:100 gave an enormous background staining and the antibody stained the nuclei of the cells (Supplementary Fig. 1B). Nuclear staining by chromogranin A is never seen with other chromogranin antibodies (for instance the well known Chr A antibody clone LK2H10 produced by Ricardo Lloyd). There was still background staining and the nuclei were stained despite using two higher dilutions [1:1500 (Supplementary Fig. 1C)] and 1:3000 (Supplementary Fig. 1D) of the antibody. In conclusion, we still lack a chromogranin A antibody that is immunoreactive to dog $\mathrm{Chr}$ A.
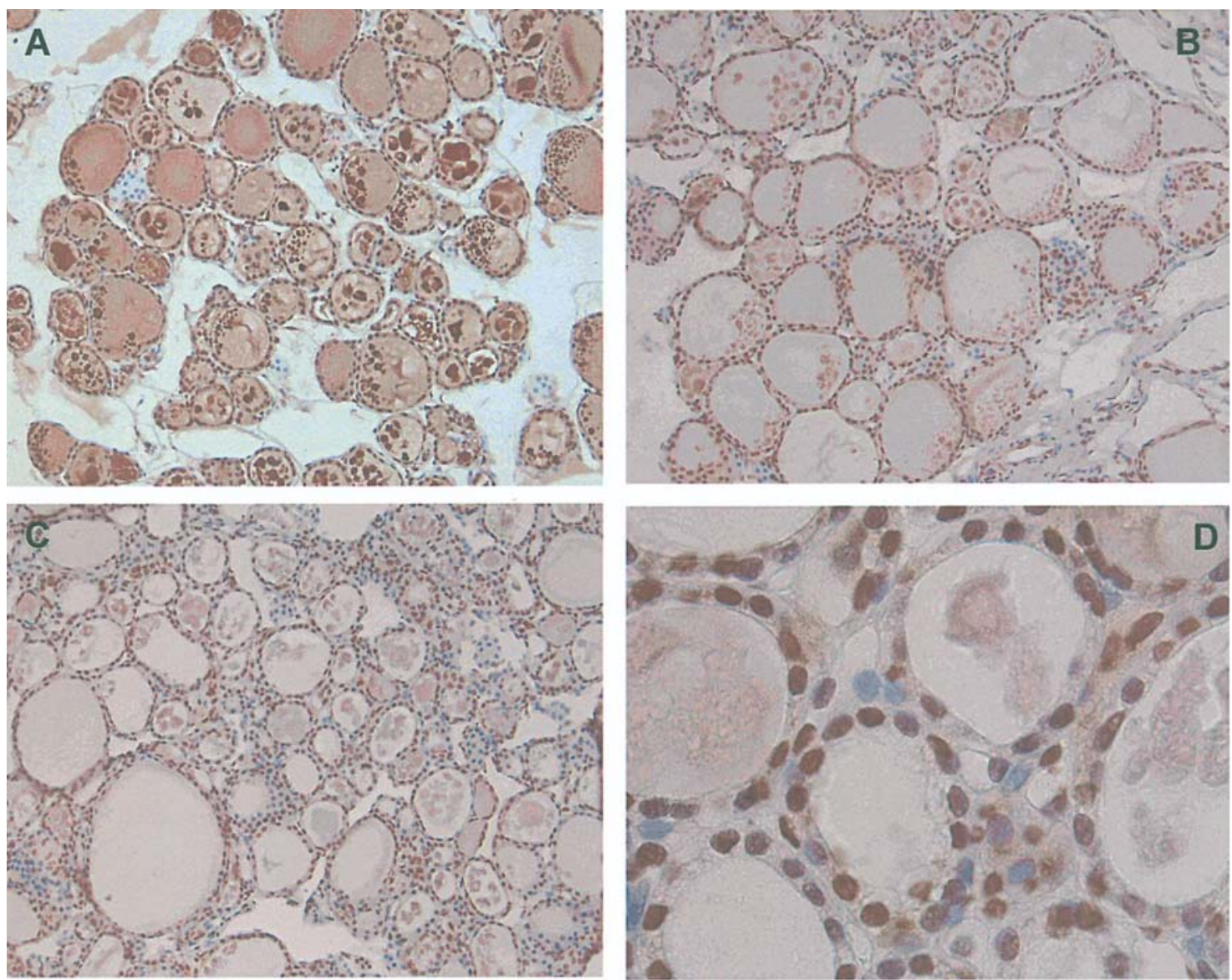

Supplementary Figure 1. Immunohistochemistry (IHC) staining for calcitonin and chromogranin A of normal dog thyroid. (A), IHC expression of calcitonin in a normal dog thyroid, x20. (B), IHC expression of chromogranin A in normal dog thyroid using 1:100 dilution of the antibody, x20. (C and D), IHC expression of chromogranin $\mathrm{A}$ in normal dog thyroid using 1:1500 dilution, $\mathrm{x} 20$. (D), IHC expression of chromogranin A in normal dog thyroid using 1:3000 dilution, $\mathrm{x} 20$ and $\mathrm{x} 100$. There was nuclei staining at this high dilution. 